The Determination of a Dynamic Cut-Off Grade for the Mining Industry

Johnson, P.V. and Evatt, G.W. and Duck, P.W. and Howell, S

2011

MIMS EPrint: 2011.98

Manchester Institute for Mathematical Sciences

School of Mathematics

The University of Manchester

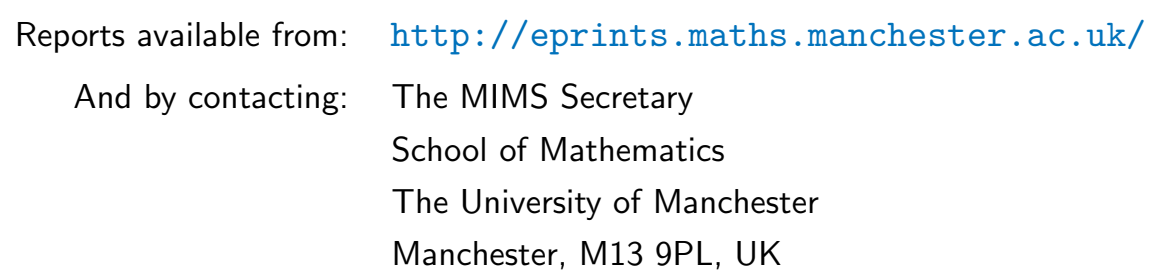

ISSN 1749-9097 


\title{
Chapter 32 \\ The Determination of a Dynamic Cut-Off Grade for the Mining Industry
}

\author{
P. V. Johnson, G. W. Evatt, P. W. Duck and S. D. Howell
}

\begin{abstract}
Prior to extraction from a mine, a pit is usually divided up into 3-D 'blocks' which contain varying levels of estimated ore-grades. From these, the order (or 'pathway') of extraction is decided, and this order of extraction can remain unchanged for several years. However, because commodity prices are uncertain, once each block is extracted from the mine, the company must decide in real-time whether the ore grade is high enough to warrant processing the block further in readiness for sale, or simply to waste the block. This paper first shows how the optimal cut-off ore grade-the level below which a block should be wasted - is not simply a function of the current commodity price and the ore grade, but also a function of the ore-grades of subsequent blocks, the costs of processing, and the bounds on the rates of processing and extraction. Secondly, the paper applies a stochastic price uncertainty, and shows how to derive an efficient mathematical algorithm to calculate and operate a dynamic optimal cut-off grade criterion throughout the extraction process, allowing the mine operator to respond to future market movements. The model is applied to a real mine composed of some 60,000 blocks, and shows that an extra $10 \%$ of value can be created by implementing such an optimal regime.
\end{abstract}

P. V. Johnson ( $₫)$ · G. W. Evatt · P. W. Duck

School of Mathematics, University of Manchester, Manchester, UK

e-mail: paul.johnson-2@manchester.ac.uk

G. W. Evatt

e-mail: geoffrey.evatt@manchester.ac.uk

P. W. Duck

e-mail: peter.duck@manchester.ac.uk

S. D. Howell

Manchester Business School, University of Manchester, Manchester, UK

e-mail: syd.howell@mbs.ac.uk 


\subsection{Introduction}

Mineral mining is a complex engineering operation, which can last for several decades. As such, significant consideration must be given to the planning and design of the operation, so that numerous engineering constraints can be met, whilst making sure the operation is economically viable. To compound the difficulty of the task, the planning and scheduling of extraction from a mine is made in the presence of uncertainties, such as the future commodity price and estimated ore-grade. These uncertainties can fluctuate on a daily basis, highlighting the different timescales upon which the mining company must base decisions: the shorter time scales governed by commodity price and realised ore-grade, and the longer time-scales governed by (amongst other things) extraction rates and processing capacities. The focus of this paper is upon one of these short time-scale decisions: whether to process the extracted material, or to waste it. The level of ore-grade which separates this decision is known as the 'cut-off grade' [7].

Prior to extraction, the planning of the extraction schedule begins with deciding an appropriate pathway (or order) through the mine. Whilst it is possible to alter the order of extraction at various points during extraction, it is generally not a particularly flexible decision, as changing an order can require moving extraction machinery, processing units, the cancellation of contracts and large overhead costs. As such, it is reasonable to assume that the pathway through the mine is fixed, but it is how one progresses, and operates, along that pathway that is variable. At this planning stage, the mine is graphically divided up into 3-D blocks, each containing its own estimated quantity of ore. The estimated ore-grade carries with it an associated uncertainty, which can have an effect upon the valuation of a mining operation [6]. However, it is the expected (estimated) ore grade level which dominates the planning of the actual pathway through the mine, as this is the best-guess in deciding the order in which the resource should be extracted. The extraction pathway is most commonly decided using software such as the Gemcom-Whittle package [15], which allows companies to construct feasible pit shapes that satisfies slope constraints on the angle of the pit, transportation needs and work-force limitations. As previously mentioned, this algorithm may be used several times throughout a mine's life, so as to ensure the mine plan is consistent with market conditions, however on a day-to-day basis the mine must take more detailed scheduling decisions in real-time.

The key real-time decision is whether or not to process the latest extracted block (e.g. by milling or electrolysis) in readiness for sale, where the block's intrinsic value varies with its ore grade and with the underlying commodity price. We define a 'cost-effective' block as one whose ore grade is high enough to pay the cash costs of processing, at the current price. However the cut-off ore gradeabove which a block should be processed-need not be set as low as the grade above which the block will be cost-effective to process. Disparity between the rate of extraction and the maximum processing capacity means that there can be an opportunity cost to processing all cost-effective material, since the small 
short-term gain of processing a low grade block could be surpassed by bringing forward the processing of more valuable blocks instead. The optimal wasting of potentially cost-effective material is the focus of this paper.

To highlight the above point, let us consider a trivial case where the mine has a stock of 3 blocks awaiting processing, extracted in order, $A, B$ and $C$, whose current market values after processing costs are $V_{A}=\$ 1, V_{B}=\$ 50$, and $V_{C}=$ $\$ 1,000$. Whilst, classically, analysis has often been indifferent to the order of processing, with enough discounting applied one can see that by an optimal cut-off criterion, it would be best to simply waste $A$ and get on with processing $B$ and $C$. This is because the value gained in processing $A$ is less than the time value of money lost in waiting to process $B$ and $C$ at a later date. This lack of consideration of the discount rate has been highlighted before as a drawback in current mine planning [14] but, as yet, little progress has been made with it.

Another consequence of an optimal cut-off grade decision is having to increase the rate of extraction of poor quality ores to keep the processing plant loaded. This is because a processing unit will typically operate at a fixed capacity, and closing (or restarting) it is a costly and undesirable operation. As such, a maximum (and minimum) possible extraction rate must be known. This clearly illustrates the link between extraction rate and the optimal cut-off grade. With this maximum possible extraction rate, one knows precisely which blocks can possibly be extracted within each period in time, and thus the decision as to which block to process next can be decided.

There have been several other approaches to mine valuation and the corresponding extraction regime. Typically these have relied upon simulation methods to capture the uncertainty of price and ore-grade $[8,9,12]$. These types of method can be extremely time consuming, with computing times of several hours [3], and can often lead to sub-optimal and incomplete results. Using these simulation techniques, optimal cut-off grades were investigated by Menabde et al. [10], although little insight into the core dynamics, performance or robustness was obtained. A similar approach is the use of genetic algorithms - a general technique commonly used by computer scientists-which are capable of calculating mine schedules whilst adhering to specified constraints upon their design [11]. Whilst the work of Myburgh and Deb [11] was suitable in calculating feasible paths, the criteria by which this particular study operated was, again, not given, and the computing time was also of the order of hours.

To make a step-change away from these methods, partial differential equations (PDEs) can be implemented to capture the full mine optimisation process, which builds on work by Brennan and Schwartz [2] and Chen and Forsyth [4]. The inclusion of stochastic ore-grade uncertainty, via PDEs has also been tested by Evatt et al. [6], which enabled mine valuations to be produced in under $10 \mathrm{~s}$ and showed that the effect on mine value of stochastic ore-grade variation is much less than the effect of stochastic price. Whilst the mathematics and numerics of this PDE approach are relatively complex at the outset, once solved, they produce highly accurate results in short times-complete with model input sensitivities. This paper extends the use of PDEs, adding a model for tactical processing 
decisions under foreseeable variations in ore grade and unforeseeable fluctuations in price. This shows that when processing capacity is constrained, the ability to maximise the value of processing by varying the cut-off ore grade can add significantly to mine value when optimally applied. By solving rapidly under a range of processing constraints, the scale of the processing plant can itself be optimised.

In Sect. 32.2 we demonstrate the underlying concepts determining the optimal cut-off decision rule, and in Sect. 32.3 we apply a price uncertainty to the model and use a contingent claims approach to derive the governing equation. We then apply the model to a mine composed of some 60,000 blocks in Sect. 32.4, to show how much extra value the running of an optimal cut-off grade regime can add to a valuation. We draw together our concluding remarks in Sect. 32.5.

\subsection{Cut-Off Grade Optimisation}

The selection of the cut-off grade criteria reduces to whether a cost-effective block should be processed or not. This is because there is the possibility a more valuable block could be brought forward in time to be processed, which otherwise would loose more time-value of money than the value gained from processing the first block. To highlight this point let us consider the order of extracted of blocks from a mine, which we (hypothetically) place in a chronologically ordered row. As we operate the processing unit of the mine, we must pass along this row and decide which blocks to process and which blocks to waste. In reality, although we know the (estimated) ore-grades of the blocks in advance, until we know for certain the market price at the time of processing we cannot know what cashflow it will generate. Yet even if we assume a constant price, we can still show how dynamic cut-off grade decision making is still required and optimal.

Consider a highly simplified mine, as shown in Fig. 32.1, which is composed of just two blocks, Block1 and Block2, with ore grades $G_{1}$ and $G_{2}$, respectively. We allow the mine to have the capacity within the rate of extraction to immediately process either the first block, Block1, or its successor, Block2. As such, the comparison is between the value of processing both blocks in order, given by $V_{12}$, or the value of only processing Block2, $V_{2}$. With a constant price, $S$, we can write down the net present value of these two (already extracted) blocks, where we shall process both,

$$
V_{12}=\left(S G_{1}-\epsilon_{P}\right)+\left(S G_{2}-\epsilon_{P}\right) e^{-r \delta t} .
$$

Here $\delta t$ is time it takes to process each block, $\epsilon_{P}$ is the cost of processing each block and the discount rate is $r$. This value must be compared to the decision to waste the first block and process only the second block, which would have value,

$$
V_{2}=\left(S G_{2}-\epsilon_{P}\right) \text {. }
$$


Fig. 32.1 Two examples of how price may effect the order in which blocks are processed so as to maximise a mines NPV. Example A is made with a low commodity price, $S=\$ 1,000 \mathrm{~kg}^{-1}$, and Example B is made with a high commodity price, $S=\$ 10,000 \mathrm{~kg}^{-1}$

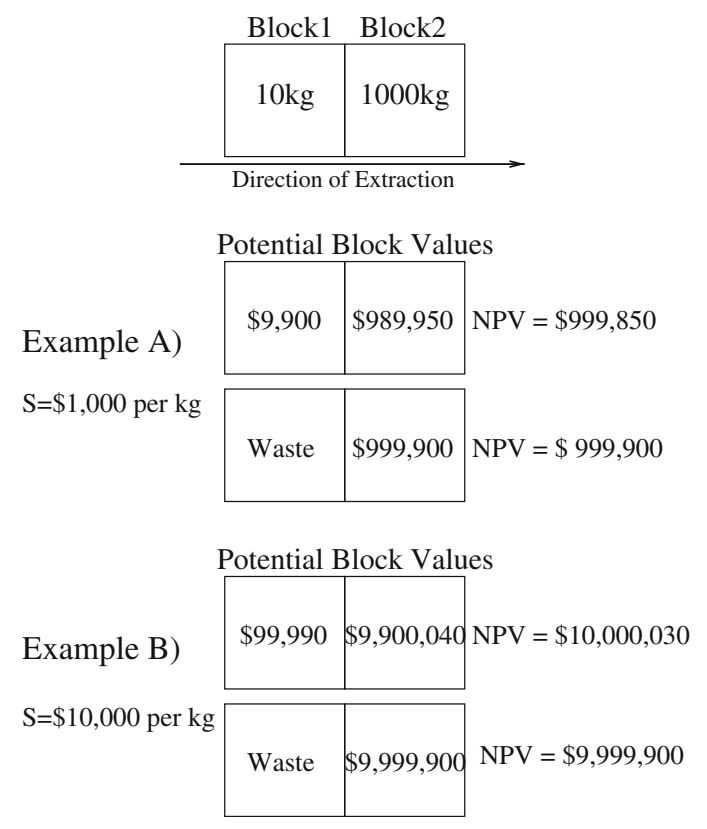

This comparison between $V_{12}$ and $V_{2}$ is one the algorithm must continually make. To demonstrate how the selection depends upon the underlying price, Fig. 32.1 shows the choices available for two different commodity prices, one high $\left(S=\$ 10,000 \mathrm{~kg}^{-1}\right)$ and one low $\left(S=\$ 1,000 \mathrm{~kg}^{-1}\right)$. These are made with prescribed parameter values

$$
r=10 \%, \quad \epsilon_{P}=\$ 100 \text { block }^{-1}, \quad \delta t=0.1 \text { year. }
$$

As can be seen, in the low-price case, Example A, it is best to process only the second block. However, in the high commodity price case, namely Example B, it is best to process both blocks. This simple example demonstrates (albeit with rather exaggerated parameter values) how the selection needs to be actively taken, and how different values of the underlying price, and discount rate, will affect the optimal cut-off decision. Another consequence of this optimal decision taking is that the mine will be exhausted earlier than might have been previously thought, since we wasted the first block and only processed the second, hence a mine owner could agree a shorter lease on this particular mine.

\subsection{Model Construction}

To create the framework for determining an optimal dynamic cut-off grade, we can make use of two distinct methods for arriving at the core equation describing the valuation, $V$. The first method follows a contingents claims approach, in which the 
uncertainty arising from the underlying price is removed by hedging away the risk via short-selling suitable quantities of the underlying resource. The second method follows the Feynman-Kac probabilistic method, as described in relation to the mining industry by Evatt et al. [5], which is the chosen method for deriving a valuation when hedging is not undertaken. This second method is also permissible when hedging does take place but a slight adjustment to the price process is required, and explained within this latter paper. Because Evatt et al. [5] already covers the derivation of the mine valuation, in the present paper we explain how the contingent claims approach can be used.

We first prescribe three state-space variables; these are the price per unit of the underlying resource in the ore $S$, the remaining amount of ore within the mine $Q$ and time $t$. We next need to define the underlying price uncertainty process, which we assume to follow a geometric Brownian motion,

$$
d S=\mu S d t+\sigma_{s} S d X_{s}
$$

where $\mu$ is the drift, $\sigma_{s}$ the volatility of $S$ and the random variable $d X_{s}$, is a standard Wiener process. We use this price process without loss of generality, since other price processes (such as mean-reverting Brownian motion) can easily be implemented by the techniques described here.

Using the contingent claims approach (see [16]) and the above notation, we may apply Ito's lemma to write an incremental diffusive change in $V$ as

$$
d V=\sigma_{s} \frac{\partial V}{\partial S} d X_{s}+\frac{\partial V}{\partial Q} d Q+\left(\frac{\partial V}{\partial t}+\frac{1}{2} \sigma_{s}^{2} \frac{\partial^{2} V}{\partial S^{2}}+\mu \frac{\partial V}{\partial S}\right) d t
$$

where we have taken powers of $(d t)^{2}$ and $(d Q)^{2}$ to be negligible. We are able to remove the $d Q$ term via the relationship between $Q$ and $t$ by specifying the rate of extraction, $q_{e}$, namely,

$$
d Q=-q_{e} d t
$$

where $q_{e}$ can be a function of all three variables, if required. This extraction rate is the function we wish to determine in our optimal cut-off regime, as it governs both how we progress through the mine and, as a consequence, which blocks we choose to waste. The rate of extraction will obviously have limitations on its operating capacity, $q_{e} \in\left[0, q_{\max }\right]$, which itself could be a function of time. The rate of extraction is closely linked to the rate of processing, which should be kept at a fixed constant, $q_{p}$. Hence $q_{\max }$ must be big enough for the processing unit to always operate at its constant capacity, $q_{p}$, i.e. there must always be enough costeffective ore-bearing material being extracted from the mine so as to meet the processing capacity. Optimal variation in the extraction rate has already been shown to produce improved valuations [7], although this was achieved without considering processing limitations or grade variation. 
With this relationship, (32.6), Eq. 32.5 can be transformed into

$$
d V=\sigma_{1} \frac{\partial V}{\partial S} d X_{s}+\left(\frac{\partial V}{\partial t}-q_{e} \frac{\partial V}{\partial Q}+\frac{1}{2} \sigma_{s}^{2} \frac{\partial^{2} V}{\partial S^{2}}+\mu \frac{\partial V}{\partial S}\right) d t
$$

To follow the conventional approach in creating and valuing risk-free portfolios we construct a portfolio, $\Pi$, in which we are instantaneously long in (owning) the mine and short in (owing) $\gamma_{s}$ amounts of commodity contracts. This defines $\Pi=$ $V-\gamma_{s} S$, such that,

$$
d \Pi=d V-\gamma_{s} d S .
$$

This portfolio is designed to contain enough freedom in $\gamma_{s}$ to be able to continually hedge away the uncertainty of $d X_{s}$, which is the standard approach in creating risk-free portfolios [1, 13]. It also implies that within a small time increment, $d t$, the value of $\Pi$ will increase by the risk-free rate of interest, minus any economic value generated and paid out by the mine during the increment. This economic value is typically composed of two parts, the first, negative, being the cost to extract, $q_{e} \epsilon_{M}$, and the second, positive, the cash generated by selling the resource content of the ore processed, $q_{p}\left(S G-\epsilon_{P}\right)$. Here $\epsilon_{M}$ is the cost of extraction per ore tonne, $\epsilon_{P}$ is the processing cost per ore tonne, and $G$ is the oregrade (weight of commodity per ore tonne). The reason why the economic functions contain the factors $q_{e}$ or $q_{p}$ is that we wish to maximise value by varying $q_{e}$ in real time, so as to maintain $q_{p}$ at its fixed bound. In turning the discrete block model into a continuous function describing the ore grade, $G$, we have assumed that blocks are small enough that they can be approximated as infinitesimal increments of volume.

As discussed in Sect. 32.2, the decision whether to process or waste the next block must be optimised. Before or after optimisation the incremental change in portfolio value may be written as

$$
d \Pi=r \Pi d t-\gamma_{S} \delta S d t-q_{p}\left(G S-\epsilon_{P}\right) d t-q_{e} \epsilon_{M} d t .
$$

By setting the appropriate value of $\gamma_{s}$ to be

$$
\gamma_{s}=\frac{\partial V}{\partial S}
$$

and substituting Eqs. (32.4), (32.7) and (32.8) into (32.9), we may write our mine valuation equation as

$$
\begin{gathered}
\frac{1}{2} \sigma_{s}^{2} S^{2} \frac{\partial^{2} V}{\partial S^{2}}+\frac{\partial V}{\partial t}-q_{e} \frac{\partial V}{\partial Q}+(r-\delta) S \frac{\partial V}{\partial S} \\
-r V+q_{p}\left(G S-\epsilon_{P}\right)-q_{e} \epsilon_{M}=0
\end{gathered}
$$

This is of the same form as that derived by Brennan and Schwartz [2], except that they added taxation terms, but did not model processing constraints or variations of ore grade. 
We next need to prescribe boundary conditions for (32.10). The boundary condition that no more profit is possible occurs either when the reserve is exhausted $Q=0$, or when a lease to operate the mine has reached its expiry date $t=T$, hence:

$$
V=0 \quad \text { on } Q=0 \quad \text { and/or } \quad t=T .
$$

Since the extraction rate will have a physical upper bound, the extraction rate and cost will not vary with $S$ when $S$ is large. This permits a far field condition of the form

$$
\frac{\partial V}{\partial S} \rightarrow A(Q, t) \quad \text { as } S \rightarrow \infty .
$$

When the underlying resource price is zero we need only solve the reduced form of Eq. 32.10 with $S=0$, which reduces to

$$
V=e^{-r t} \int_{0}^{T} q_{e} \epsilon_{M}(z) e^{r z} d z .
$$

This completes the determination of our core equation, and its boundary conditions. We can now define the optimising problem which we wish to solve: we must determine the optimal extraction rate, $q_{e}^{*}$, at every point in the state space which maximises the value $V$, which satisfies Eq. 32.10, with $q_{e}=q_{e}^{*}$, subject to the defined boundary conditions. Problems of this type may be solved numerically using finite-difference methods, in particular the semi-Lagrangian numerical technique (see [4] for further details). All results in this paper have been thoroughly tested for numerical convergence and stability.

We must now show how the optimal $q^{*}$ and its corresponding cut-off grade is to be incorporated into the maximisation procedure.

\subsection{Example Valuation}

We now apply our optimal cut-off grade model to a real mine of some 60,000 blocks, whose block by block ore-grade and sequence of extraction were supplied by Gemcom Software International. This mine has an initial capital expenditure of some $\$ 250 \mathrm{~m}$. We were also supplied with a fixed reference price $S_{r e f}$, for us to compare valuations with. We ourselves assumed a maximum extraction rate of five times the processing rate, which is broadly realistic, and it restricts the mine to wasting no more than $80 \%$ of any section of costeffective ore (if one can increase the extraction rate fivefold, then it is possible to waste four blocks and process the fifth). The other parameter values we were supplied are 


$$
\begin{aligned}
& r=10 \% \text { year }^{-1}, \quad \delta=10 \% \text { year }^{-1}, \quad \sigma_{s}=30 \% \text { year }^{-\frac{1}{2}}, \\
& S_{\text {ref }}=\$ 11,800 \mathrm{~kg}^{-1}, \quad \epsilon_{P}=\$ 4 \text { tonne }^{-1}, \quad \epsilon_{e}=\$ 1 \text { tonne }^{-1}, \\
& Q_{\text {max }}=305,000,000 \text { tonnes }, \quad q_{p}=20,000,000 \text { tonnes year }^{-1} .
\end{aligned}
$$

Whilst the ore-grade is quite volatile, it was shown in Evatt et al. [6] that a suitable average of the estimated grade quality could be used without any sizeable alteration in the valuation, as one would expect, since the same volume of ore is available sold whether one takes average values or not. Using this average, Fig. 32.2 shows the economic worth throughout extraction for each part of the mine, where we have assumed the price to remain at its prescribed reference price, $S_{\text {ref }} G-\epsilon_{P}$. This highlights how the grade varies through the extraction process, and it is with reference to this grade variation that we shall compare the regions where it is optimal to speed up extraction and consequently waste certain parts of the ore body.

\subsubsection{Results}

For the example mine, we first calculate and compare two different valuations made with and without the optimal cut-off criterion. Figure 32.3 shows two sets of valuations: the lower pair (straight lines; one dashed, one solid) shows the valuations made assuming a constant price $\left(\sigma_{s}=0 \%\right)$, and the upper pair (curved lines; one dashed, one solid) shows the effect of including both price uncertainty $\left(\sigma_{s}=30 \%\right)$ and the option to abandon the mine when the valuation becomes negative-which is a standard option to include in a reserve valuation [2]. In each pair of lines the lower, dotted lines show valuation without a cut-off regime, and the higher, solid lines show valuation with the optimal cut-off regime. The optimal cut-off regime increases the mine valuation by up to $10 \%$, with increasing benefit

Fig. 32.2 Given a block ordering in the mine, the average standardised grade value is the cash value of ore (against reference price) minus processing costs per tonne of ore. This data was supplied by Gemcom Software International

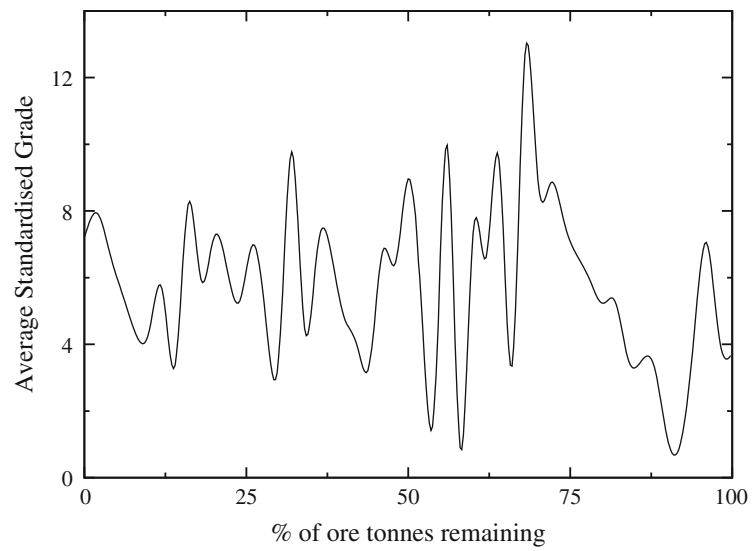


at higher prices. This may seem surprising, but although the mine is always more profitable at higher prices, the opportunity cost of not allocating the finite processing capacity to the best available block does itself grow.

An obvious question which arises from this analysis is how do we decide which ore-grades we should waste, and what is the corresponding rate of extraction to achieve this? Given the mine operator will know at each point in time what the current underlying price is, they can look at the corresponding slice through the 3 -D surface of the optimal cut-off grade, and see for which regions in $t$ and $Q$ they would waste ore and increase the rate of extraction. With this we can refer back to the corresponding grade of Fig. 32.2 and easily calculate what these grades actually are. For example, by looking at the closed regions of Fig. 32.4 we can see the optimal cut-off grades for two different commodity prices, $S=100 \%$ (top) and $S=200 \%$ (bottom) of the reference price. The points at where it is optimal to increase the rate of extraction is given by the segments where the closed regions (bounded by the thin line) intersect with the optimal extraction trajectory (bold line). In the two examples of Fig. 32.4, both appear to correspond to a standardised cut-off grade (Fig. 32.2) of around 2 units. The optimal rate of extraction is given by the gradient of the bold line, where the trajectory is calculated by integrating (32.6) for a given extraction regime. The difference between the dotted line (trajectory for the no cut-off situation), and the thick straight line of the optimal cut-off regime therefore gives an indication of the total amount of ore wasted.

Finally, Fig. 32.5 shows how the NPV depends upon the expected expiry time for extraction if one operates an optimal cut-off regime (solid line) or not (dotted line). If the mine chooses the optimal regime, the maximum NPV occurs just after 14 years, as opposed to the life of the mine being maximal at mine exhaustion at 15 years (as it is with no cut-off). This is a consequence of an optimal cut-off grade regime, in which the mine will occasionally increase its extraction rate from the (originally) planned level due to market fluctuations, thereby reaching the final pit shape in a shorter time.

Fig. 32.3 NPV of the mine against percentage of reference price for two different sets of valuations. The two lower lines (straight lines; one dashed, one solid) are for a constant price while the two upper lines (curved lines; one dashed, one solid) include price volatility and the abandonment option. NPV for the optimal cut-off regime is shown by solid lines, and no cut-off by dashed lines

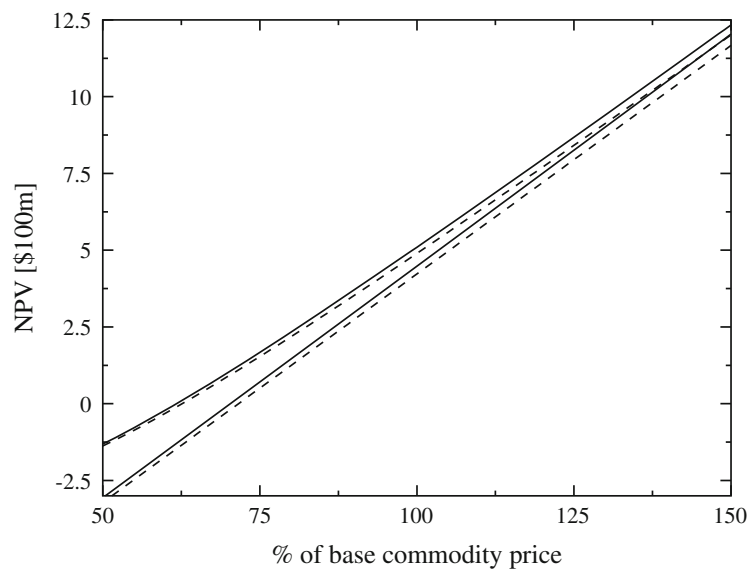


Fig. 32.4 Graphs showing the optimal cut-off regions for an extraction project for two different price levels, medium (top), and high (bottom). The closed regions contained within the thin solid lines show where ore is wasted and the extraction rate is increased. The dashed line represents the one realisation of a trajectory followed with no cut-off, while the thick solid line represents the realisation of the trajectory followed with optimal cut-off
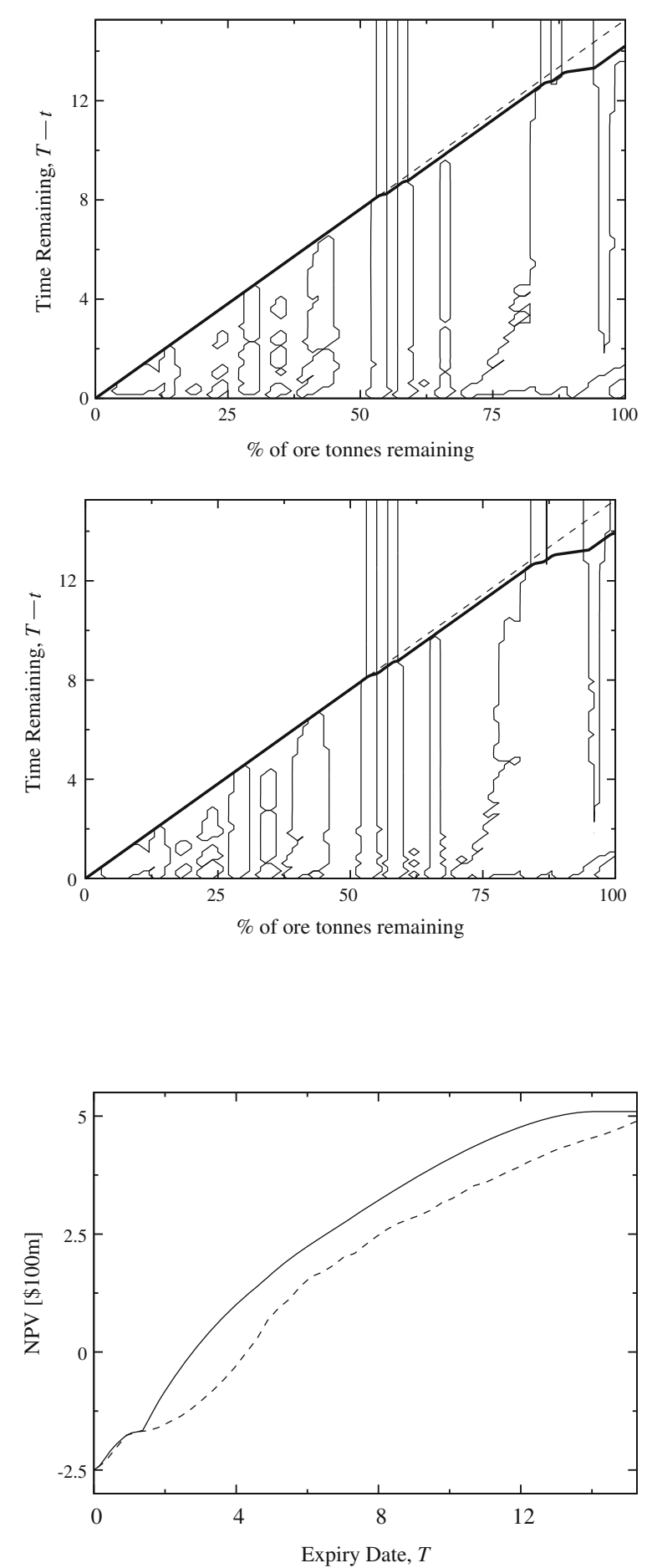

Expiry Date, $T$
Fig. 32.5 The NPV of the mine against time remaining on the option on the mine given that $100 \%$ of the mine is present. The solid line is with optimal cut-off, dashed without 


\subsection{Conclusions}

This paper has shown how to solve and optimise a (relatively) short time-scale mining problem, known as a dynamic cut-off grade, which is the continuous decision of whether to process extracted ore or not. This was achieved in the presence of price uncertainty. We have described how the partial differential equation model can be derived via two distinct methods, either by a contingent claims approach, when continuous hedging is present, or by the Feynman-Kac method. Using this model, we have shown how to determine and operate a optimal dynamic cut-off grade regime. As such, we have valued the 'option' to process or not to process under uncertainty, allowing the mine owner to react to future market conditions.

With our given example, the option adds around $10 \%$ to the expected NPV of an actual mine of 60,000 blocks. One natural extension of this work will be to allow for the cut-off grade to remain fixed for discrete periods of time, thus allowing mine operators to not have to continually alter their rate of extraction due to market changes.

\section{References}

1. Black F (1976) The pricing of commodity contracts. J Financial Econ 3:167-179

2. Brennan MJ, Schwartz ES (1985) Evaluating natural resource investments. J Business 58(2):135-157

3. Caccetta L, Hill SP (2003) An application of branch and cut to open pit mine scheduling. J Global Optim 27:349-365

4. Chen Z, Forsyth PA (2007) A semi-Lagrangian approach for natural gas storage valuation and optimal operation. SIAM J Sci Comput 30(1):339-368

5. Evatt GW, Johnson PV, Duck PW, Howell SD, Moriarty J (2010) The expected lifetime of an extraction project. In: Proceedings of the Royal Society A, Firstcite. doi:10.1098/rspa. 2010.0247

6. Evatt GW, Johnson PV, Duck PW, Howell SD (2010) Mine valuations in the presence of a stochastic ore-grade. In: Lecture notes in engineering and computer science: proceedings of the World Congress on engineering 2010, vol III, WCE 2010, 30 June-2 July, 2010, London, UK, pp 1811-1866

7. Johnson PV, Evatt GW, Duck PW, Howell SD (2010) The derivation and impact of an optimal cut-off grade regime upon mine valuation. In: Lecture notes in engineering and computer science: proceedings of the World Congress on engineering 2010, WCE 2010, 30 June-2 July, 2010, London, UK, pp 358-364

8. Jewbali A, Dimitrakopoulos R (2009) Stochastic mine planning-example and value from integrating long- and short-term mine planning through simulated grade control. Orebody modelling and strategic mine planning, 2nd edn. The Australasian Institute of Mining and Metallurgy, Melbourne, pp 327-333

9. Martinez LA (2009) Designing, planning and evaluating a gold mine project under in-situ metal grade and metal price uncertainties. Orebody modelling and strategic mine planning, 2nd edn. The Australasian Institute of Mining and Metallurgy, Melbourne, pp 225-234

10. Menabde M, Foyland G, Stone P, Yeates GA (2004) Mining schedule optimisation for conditionally simulated orebodies. In: Proceedings of the international symposium on orebody modelling and strategic mine planning: uncertainty and risk management, pp 347-52 
11. Myburgh C, Deb K (2010) Evolutionary algorithms in large-scale open pit mine scheduling. In: Proceedings of the 12th annual conference on genetic and evolutionary computation, pp 1155-1162

12. Ramazan S, Dimitrakopoulos R (2007) Stochastic optimisation of long-term production scheduling for open pit mines with a new integer programming formulation. Orebody modelling and strategic mine planning. The Australasian Institute of Mining and Metallurgy, Melbourne, pp 385-391

13. Schwartz ES (1997) The stochastic behavior of commodity prices: implications for valuation and hedging. J Finance LII(3):923-973

14. Tolwinski B, Underwood R (1996) A scheduling algorithm for open pit mines. IMA J Math Appl Bus Ind 7:247-270

15. Whittle D, Cahill J (2001) Who plans mines? In: Strategic mine planning conference, Perth, WA, pp 15-18

16. Wilmott P, Howison S, Dewynne J (1995) The mathematics of financial derivatives. Cambridge University Press, Cambridge 